\title{
Oral manifestations of viral infections
}

Van Heerden WFP, BChD, MChD (Oral Path), FC Path (SA) Oral Path, PhD, DSc Department of Oral Pathology and Oral Biology, University of Pretoria

Correspondence to: Prof Willie van Heerden, e-mail: wvheerd@medic.up.ac.za

\section{Abstract}

Examination of the oral cavity is an integral part of every clinical examination. The clinical presentation of various viral conditions is discussed in this article and will provide the family practitioner with an up to date guide to better diagnosis and management.

SA Fam Pract 2006;48(8): 20-24

\section{Introduction}

Viral infections of the oral mucosa are frequently encountered in general practice. The clinical diagnosis of these lesions can sometimes be confusing due to similar clinical presentations. The clinical presentation, diagnosis and appropriate management of common viral infections of the oral mucosa are discussed.

\section{Herpes simplex virus Primary herpetic gingivostomatitis}

The infection that accompanies primary herpetic gingivostomatitis is usually subclinical in early childhood and only a small percentage of patients develop an acute primary infection. This usually occurs in older children and consists of fever, malaise, headache, cervical lymphadenopathy and a vesiculo-ulcerative eruption on the peri-oral skin, vermilion or any intra-oral mucosal surface. ${ }^{1}$ The vesicles, which are 2 to $3 \mathrm{~mm}$ in diameter, rupture, leaving painful ulcers that heal without scarring after seven to ten days. The gingivae are swollen and reddish due to a general inflammation (see Figure 1). The virus then migrates to the trigeminal ganglion, where it remains latent. In more affluent countries with better living conditions and less overcrowding, many young adults do not acquire the infection during childhood. They are at risk of developing a symptomatic infection as an adult, usually presenting as a pharyngotonsillitis, with constitutional symptoms of fever, malaise and headaches. In the case of cervical lymphadenopathy, the vesicles and ulcers on the tonsils and posterior pharynx can resemble infectious mononucleosis or a streptococcal sore throat infection. Primary infection in an immunocompromised adult can be life threatening, with disseminated disease, or it may present with extensive nonhealing oral ulceration. ${ }^{2}$

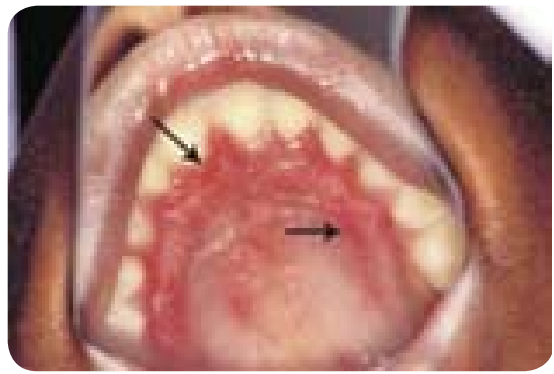

Figure 1: Primary herpetic gingivostomatitis resulting in painful erythematous palatal gingivae (arrows)

\section{Secondary herpes labialis}

Around 15 to $30 \%$ of the community is affected by episodes of secondary herpes simplex lesions (herpes labialis). Common colds, influenza, fever, UV exposure, menstruation, emotional upset, stress and anxiety predispose the patient to recurrent infection, as these cause reactivation of the virus, which subsequently migrates along one of the sensory divisions of the trigeminal nerve. The lesions are most often seen at the mucocutaneous junction of the lip or peri-oral skin (see Figure 2). A burning sensation usually precedes the development of a small cluster of vesicles. These vesicles enlarge, coalesce, ulcerate and become crusted before healing within 10 days.

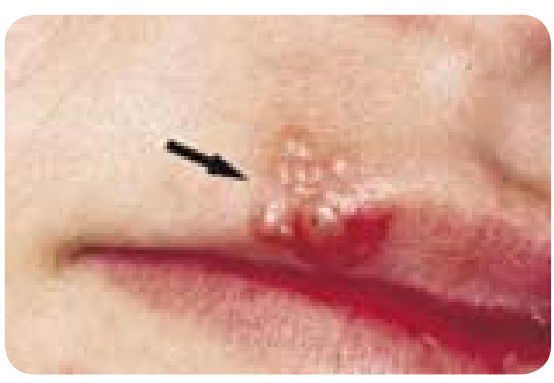

Figure 2: Herpes labialis, consisting of a cluster of vesicles on the vermillion (arrow).

\section{Diagnosis and management}

The clinical features are usually sufficient to diagnose these conditions. The differential diagnosis of primary herpetic gingivostomatitis includes recurrent aphthous ulceration, which forms ulcers on non-keratinised oral mucosa without a vesicle phase.

There currently is no drug available to prevent the migration of the herpes virus to the trigeminal ganglion after primary infection. Acyclovir is a potent drug and may be life saving for herpetic encephalitis and disseminated infection, especially in those individuals who are immuno-compromised. It will accelerate healing if used sufficiently early in the disease stage. Bed rest, fluids and a soft diet, with antipyretics for fever, are recommended for primary herpetic gingivostomatitis. Preventative therapy, such as using sun block, is the management of choice for herpes labialis, while antiviral drugs such as acyclovir are only useful if applied at the prodromal stage before the development of vesicles. ${ }^{3}$ Patients must be discouraged from touching the lesions 
in order to reduce the risk of spreading the infection to other sites. Systemic acyclovir may be used for immunocompromised individuals. ${ }^{1}$

\section{Varicella-zoster virus}

Chickenpox (varicella) results from primary infection, while reactivation of the virus is known as herpes zoster (shingles). Intra-oral vesicles of varicella, when present, are seen on the tongue, buccal mucosa, gingival, palate and oropharynx. They generally are not very painful. Following the primary infection, the virus is transported via the sensory nerves to the dorsal spinal ganglia or trigeminal ganglion, where it remains latent.

A number of predisposing factors can lead to a recurrence of the infection in the tissue supplied by the sensory nerve. Conditions leading to herpes zoster are usually those that cause immunosuppression, such as cytotoxic drugs, radiation, internal malignancies, malnutrition, old age, and alcohol and substance abuse. Occasionally, dental manipulation in a localised area can lead to reoccurrence. The first signs of herpes zoster are pain and tenderness in the dermatome corresponding to the affected sensory ganglion. In the head and neck area, vesicles form on one side of the face or in the oral mucosa in one of the divisions of $\mathrm{N}$. trigeminus. These unilateral vesicles form clusters with areas of surrounding erythema, ending abruptly in the midline (see Figure 3). The vesicles ulcerate and form pustules within three to four days. A crust lesion then forms, and healing takes place within seven to ten days. These lesions often heal with scarring and areas of hypo/hyperpigmentation may be seen. When the $\mathrm{N}$. facialis and $\mathrm{N}$. auditorius are involved (via the geniculate ganglion), facial paralysis, vesicles on the external ear, tinnitus, deafness and vertigo may follow. This is known as Ramsay-Hunt syndrome. ${ }^{4}$

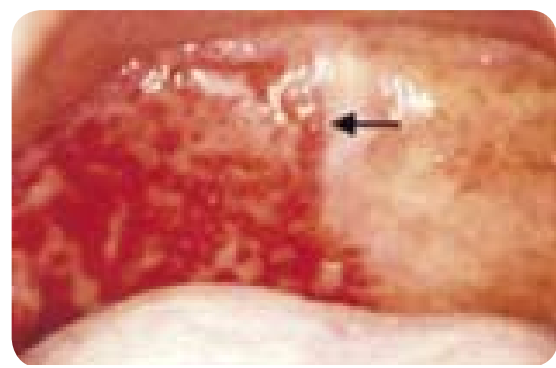

Figure 3: Herpes zoster, presenting with multiple ulcers on the palate. Note the abrupt ending of the ulcers at the midline (arrow).
Spontaneous bone necrosis of the mandible, occurring during an acute attack of herpes zoster, has been seen in immunocompromised patients. ${ }^{5}$ The patients present with the unilateral vesicles of herpes zoster, with subsequent unilateral exfoliation of the teeth in the necrotic mandible (see Figure 4).

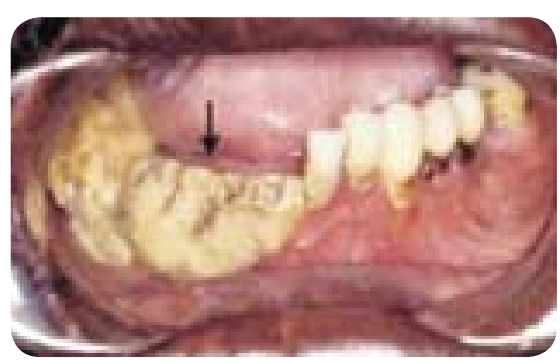

Figure 4: Unilateral necrosis of the mandible (arrow) in an HIV-positive man with a CD4 count of less than 200

A residual complication of herpes zoster is post-herpetic neuralgia. It occurs in $10 \%$ of patients with herpes zoster and affects the trigeminal nerve, most commonly the ophthalmic division. There is persistent, unilateral pain in the affected area. A history of previous skin lesions and possible scarring may aid in the diagnosis. The pain is not paroxysmal, as is seen in trigeminal neuralgia, although it may be just as severe.

\section{Diagnosis and management}

The clinical picture is often distinctive. Herpes zoster may be confused with recurrent Herpes simplex virus infection. Herpes zoster has a longer duration, a more severe prodromal phase, unilateral vesicles and ulceration, with abrupt ending at the midline and postherpetic neuralgia

The treatment is supportive and symptomatic, with topical or systemic antipruritics and analgesics that do not contain aspirin. A high dose of oral acyclovir (800 mg five times daily for seven days) is recommended for treating both primary and recurrent infections in immunocompromised patients. ${ }^{6}$

\section{Coxsackie virus} Herpangina

Herpangina affects children, mainly during summer, and is characterised by a sudden onset of malaise, fever and a sore throat. Patients present with vesicles, ulcerations, and diffuse erythema on the soft palate, fauces and tonsillar areas (see Figure 5). The systemic symptoms settle in two to three days and the ulcers heal in seven to ten days.

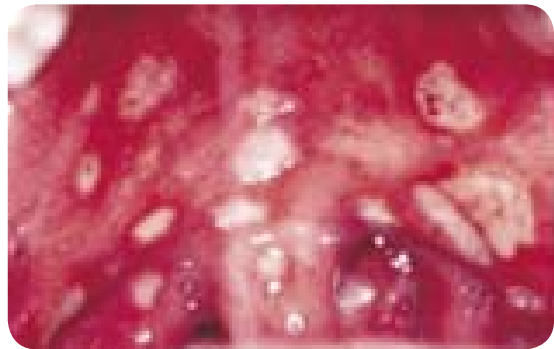

Figure 5: Herpangina, presenting with multiple ulcers on the soft palate, uvula and fauces.

\section{Hand, foot and mouth disease}

Hand, foot and mouth disease is most commonly seen amongst children aged one to five years. In $75 \%$ of cases it presents with an eruption of vesicles on the palms of the hands and on the feet. Occasional vesicles may also be found on the proximal extremities and buttocks. There are also vesicles in the anterior part of the mouth (see Figure 6). An associated low-grade fever and malaise are usually present.

\section{Diagnosis and management}

The clinical features of Coxsackie virus are distinctive. The distribution of the lesions of herpangina differentiates it from primary herpetic gingivostomatitis, which affects the gingivae, whereas herpangina is an oropharyngitis. The systemic symptoms differentiate it from recurrent aphthous ulceration. The vesicles also help to distinguish herpangina from streptococcal pharyngitis

In the case of hand, foot and mouth disease, the cutaneous vesicles can resemble chickenpox; however, in chickenpox the vesicles usually start on the face and trunk and then spread to the extremities. Involvement of the palms and soles is rare in chickenpox infections. Oral lesions are also rare in chickenpox.

Herpangina and hand, foot and mouth disease are self-limiting, of short duration and need no treatment. Symptomatic treatment is indicated in very painful cases of herpangina, in which case non-aspirin antipyretics and topical anaesthetics can be used.

\section{Human papillomavirus Focal epithelial hyperplasia (Heck's disease)}

Heck's disease presents with multiple asymptomatic, slightly elevated, mucosa-coloured, smooth-surfaced nodules that occur on the labial or buccal mucosa gingivae or tongue of children (see Figure 7). Individual lesions tend to be small $(0.3$ to $1 \mathrm{~cm})$, but they frequently cluster and coalesce, giving the 
mucosa a cobblestone or fissured surface. Most lesions are found in children, although they occasionally can be found in older age groups. They usually regress spontaneously with age.

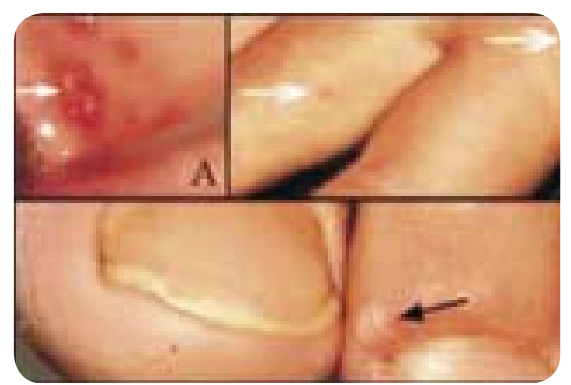

Figure 6: Patient with hand, foot and mouth disease, presenting with $(A)$ intra-oral ulcerations (arrow) secondary to vesicles, with (B) small vesicles on his palm and fingers (arrows) and (C) a vesicle on the toe (arrow) is clearly visible.

\section{Squamous cell papilloma and ver- ruca vulgaris}

Squamous papilloma is a slow-growing, solitary, painless, cauliflower-like lesion with branched finger-like processes (see Figure 8). It can occur at any age, but is most commonly diagnosed in the age group 30 to 50 years. It may initially grow rapidly, but seldom grows beyond $5 \mathrm{~mm}$ in diameter. Although any site of the oral cavity may be affected, the tongue, lips and soft palate are common locations. The lesion will range from reddish to white, depending on the degree of surface keratinisation.

Verruca vulgaris is a very common childhood infection. Oral lesions usually arise from autoinoculation, most commonly on the labial mucosa, tongue and gingiva. It presents as a slow-growing, painless, exophytic lesion that consists of sharp, finger-like processes.

\section{Condyloma accuminatum}

Condyloma accuminatum is usually present on the genitals, although it may be found in the oral cavity. Oral lesions are predominantly transmitted through oral-genital sexual contact. It consists of multiple cauliflower-like lesions, some of which are larger than $0.5 \mathrm{~cm}$. They may become as large as $3 \mathrm{~cm}$ in size. The most common intra-oral sites are the labial mucosa and the lingual frenum and soft palate.

\section{Diagnosis and management}

No treatment is usually required for focal epithelial hyperplasia, as the nodules undergo spontaneous regression during puberty. Lesions that cause aesthetic problems or are chronically injured can be removed surgically.

It is often impossible to distinguish between verruca vulgaris and squamous cell papilloma of the oral mucosa. Excision of both lesions is the treatment of choice.

Condyloma accuminatum should not be confused with focal epithelial hyperplasia. It may be difficult to distinguish from squamous cell papilloma, which is usually single and not larger than $0.5 \mathrm{~cm}$. Excision is the treatment of choice. The presence of these lesions in children should raise the possibility of sexual abuse, although on rare occasions they may be transmitted by nonsexual contact. ${ }^{7}$

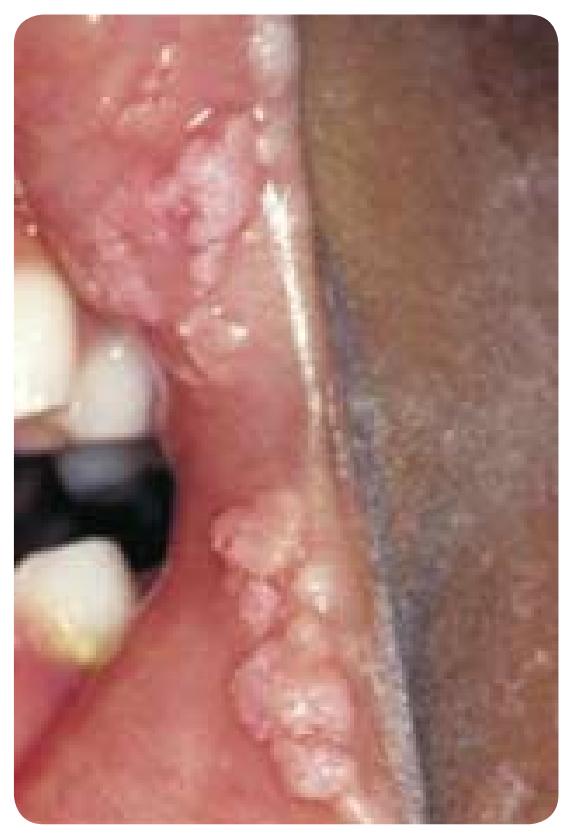

Figure 7: Focal epithelial hyperplasia at the mucosa of the corner of the mouth, consisting of multiple, slightly elevated, mucosacoloured nodules.

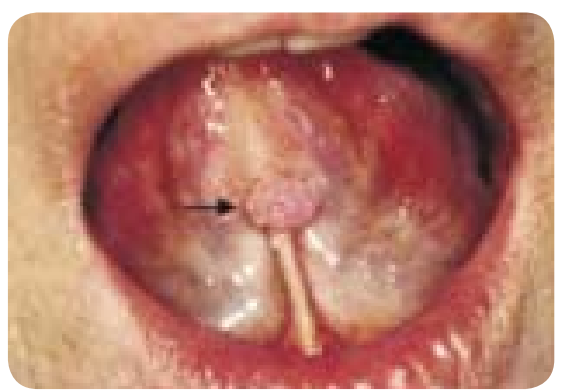

Figure 8: Squamous papilloma presenting as a single cauliflower-like lesion (arrow) on the lingual frenum in an adult.

\section{Measles}

Measles, a paramyxovirus infection, is most commonly seen in children as an acute febrile illness and erythema- tous maculopapular skin rash. The incidence of measles has decreased dramatically in many communities due to successful vaccination programs although it has re-emerged in unvaccinated children, mostly amongst children of poor rural communities. Following an incubation period of 10 to 12 days, a prodromal phase consisting of fever, malaise, conjunctivitis, cough and coryza is seen. This is followed by a generalized exanthematous skin rash. The oral manifestation of measles is known as Koplik's spots. These occur early in the course of the infection and often precede the skin rash by 1 to 2 days. Koplik's spots are white-red macules on that appear on the buccal and labial mucosa (see Figure 9). These macules represent foci of epithelial necrosis. ${ }^{8}$

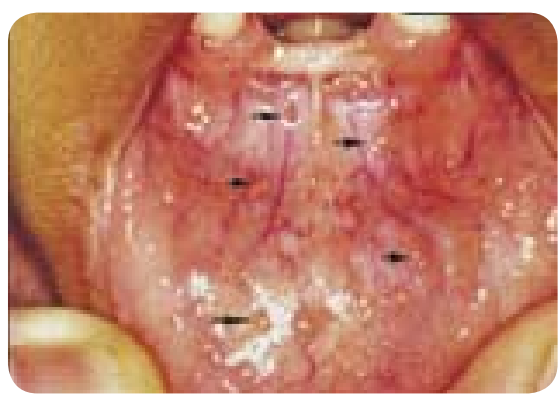

Figure 9: Koplik's spots on the labial mucosa present as numerous small macules (arrows).

\section{Diagnosis and management}

Diagnosis is based on the clinical features and history. Laboratory confirmation is possible for atypical cases. Supportive therapy including bed rest, fluids, adequate diet and non-aspirin antipyretics are recommended for symptomatic relief.

\section{Human immunodeficiency virus}

Although no oral lesions are found as a direct result of HIV infection itself, a range of HIV-associated oral lesions, some with a viral aetiology, have been described.

\section{Oral hairy leukoplakia}

This presents as a white, vertically corrugated, non-removable lesion on the lateral or ventral margin of the tongue (see Figure 10). The surface might be non-corrugated if it is seen on the inferior surface of the tongue or in the buccal mucosa. This lesion is caused by the Epstein-Barr virus and has no premalignant potential. It has also been reported in HIV-negative persons 
in association with immunosuppresive therapy. It must be differentiated from chronic hyperplastic candidiasis or leukoplakia (a potentially malignant lesion). A biopsy must be performed to establish a reliable diag-nosis. Once the diagnosis has been made, no treatment for the lesion is necessary.

\section{Kaposi's sarcoma}

Kaposi's sarcoma is characterised by erythematous or violaceous plaquelike lesions that develop into tumorous growths over time (see Figure 11). These larger lesions may become ulcerated and painful and may interfere with function. Kaposi's sarcoma is predominantly seen in the palate or on the attached gingivae, but can appear on other mucosal sites. It has been demonstrated that Kaposi's sarcoma is caused by infection with human herpes virus 8. The clinical diagnosis should be confirmed by biopsy, as several other lesions may have similar clinical presentations. This is especially true for early lesions that may have similar clinical features as vascular malformations, bacillary angiomatosis and even well differentiated angiosarcoma. A variety of treatment options are available varying from intralesional injection of chemotherapeutic or sclerosing agents to surgery or radiation. Regression of Kaposi's sarcoma has been reported in patients receiving combination antiretroviral therapy.

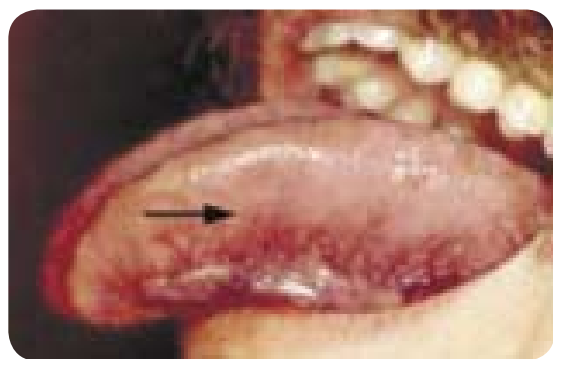

Figure 10: Oral hairy leukoplakia on the lateral border of the tongue, with extension onto the smooth ventral aspect of the tongue (arrow).

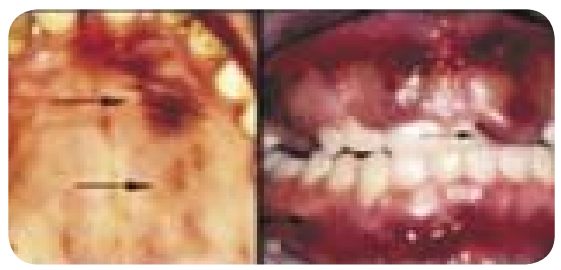

Figure 11: Kaposi's sarcoma of the palate (arrows) in (A) the early plaque stage and in (B) the more advanced tumorous phase on the gingiva (arrows).

\section{Conclusion}

Examination of the oral cavity should routinely be performed as the oral mucosa is often the first site affected by viral infections. A thorough medical history together with a detailed examination will result in an accurate diagnosis for the majority of viral lesions affecting the oral cavity, resulting in appropriate patient care. Persistent lesions, especially if ulcerated, should be biopsied to exclude the possibility of a non-infective, more serious aetiology. Biopsies or other laboratory investigations should also be performed if a clinical diagnosis can not be established. This is especially true in the setting of HIV/AIDS that can influence the typical clinical features of viral infections.

\section{See CPD Questionnaire, page 44}

\section{P This article has been peer reviewed}

\section{References}

1. McCullough MJ, Savage NW. Oral viral infections and the therapeutic use of antiviral agents in dentistry. Aust Dent J 2005;50:S31-5.

2. Schubert MM. Oral manifestations of viral infections in immunocompromised patients. Curr Opin Dent 1991;1:384-97

3. Raborn GW, Grace MG. Recurrent herpes simplex labialis: selected therapeutic options. J Can Dent Assoc 2003;69:498-503.

4. Sweeney CJ, Gilden DH. Ramsay-Hunt syndrome. J Neurol Neurosurg Psychiatry 2001;71:149-54.

5. Van Heerden WF, McEachen SE, Boy SC. Alveolar bone necrosis and tooth exfoliation secondary to herpes zoster in the setting of HIV/AIDS. Aids 2005; 19:2183-4.

6. Reusser P. Management of viral infections in immunocompromised cancer patients. Swiss Med Wkly 2002;132:374-8.

7. Kui LL, Xiu HZ, Ning LY. Condyloma acuminatum and human papilloma virus infection in the oral mucosa of children. Pediatr Dent 2003;25:149-53.

8. Neville BD, Damm DD, Allen CM, Bouquot JE. Oral and Maxillofacial Pathology, $2^{\text {nd }}$ ed. WB Saunders Co, Philadelphia 2002. 